Check for updates

Cite this: J. Mater. Chem. C, 2020 8, 550

Received 29th October 2019 Accepted 17th November 2019

DOI: 10.1039/c9tc05913j

rsc.li/materials-c

\section{A stretchable and compressible ion gel based on a deep eutectic solvent applied as a strain sensor and electrolyte for supercapacitors $\dagger$}

\author{
Shu Hong, (D) ab Yang Yuan, ${ }^{a}$ Chaozheng Liu, (D) ${ }^{a}$ Weimin Chen, (D) ${ }^{a}$ Ling Chen, ${ }^{a}$ \\ Hailan Lian (iD) *a and Henrikki Liimatainen (D) ${ }^{b}$
}

\begin{abstract}
An ion gel based on a deep eutectic solvent (DES) was fabricated using free radical polymerization of an acrylamide monomer in a choline chloride-urea-glycerol ( $\mathrm{ChCl}-\mathrm{U}-\mathrm{G}$ ) system. The mechanical properties of the ion gel were reinforced by dispersing cellulose pulp in the DES without losing its conductivity. The obtained ion gel was flexible and strong, and it acted like an elastomer. Its excellent mechanical properties can be ascribed to the formation of a coherent hydrogen bond network between the DES and the polymer matrix. The ion gel was successfully applied as a sensor to monitor the grasping motion of the hand, which provides a new approach for designing green electronics (e.g., for electronic skin). The ion gel was also applied as a solid-state electrolyte in a symmetric supercapacitor with a wide operating voltage window of $2 \mathrm{~V}$ and a high specific capacitance of $161.8 \mathrm{~F} \mathrm{~g}^{-1}$ at a current density of $0.2 \mathrm{~A} \mathrm{~g}^{-1}$. It also exhibited a high energy density of $22.47 \mathrm{~W} \mathrm{~h} \mathrm{~kg}^{-1}$ at a power density of $0.11 \mathrm{~kW} \mathrm{~kg}^{-1}$ and outstanding cyclability (95.3\% capacitance retention after 2000 cycles at $1.0 \mathrm{~A} \mathrm{~g}^{-1}$ ). Moreover, the main components of the prepared multifunctional ion gel are biocompatible, cost-effective, and environmentally friendly, offering a new route for the fabrication of green and adaptable structures for electronics.
\end{abstract}

\section{Introduction}

Due to the rapid progress of flexible and wearable electronic devices, it is critical to develop stretchable power supplies. ${ }^{1-3}$ For this purpose, supercapacitors offer distinctive advantages, such as a simple device structure, high power density, and excellent charge-discharge cyclability, in comparison to batteries. ${ }^{4-6}$ Supercapacitors can be simply constructed by inserting two high-surface-area carbon electrodes in salt water. Double layer supercapacitors only store charges via electrostatic ion absorption at the liquid-solid interface. ${ }^{5}$ Significant research efforts have focused on developing flexible electrode materials. ${ }^{6-9}$ However, it is also crucial to develop flexible electrolyte materials with appropriate conductivity and elasticity to produce flexible energy storage materials. Since stretchability and compressibility are typical features of the new generation of flexible devices. ${ }^{10}$ Both the electrode and electrolyte should meet this requirement to achieve the goal.

\footnotetext{
${ }^{a}$ College of Materials Science and Engineering, Nanjing Forestry University, Nanjing, 210037, China. E-mail: lianhailan@njfu.edu.cn

${ }^{b}$ Fibre and Particle Engineering Research Unit, University of Oulu, P.O. Box 4300, 90014 Oulu, Finland

$\dagger$ Electronic supplementary information (ESI) available. See DOI: 10.1039/ c9tc05913j
}

Traditional solid-state and flexible electrolytes are poly(vinyl alcohol) (PVA)-based gels. They are not feasible for flexible energy storage materials because of their intrinsic leakage problem. ${ }^{11-15}$ Therefore, polymeric hydrogels have attracted much scientific interest due to their versatile properties. ${ }^{16-18}$ However, when not combined with other materials, polymeric hydrogels are often mechanically weak or brittle. ${ }^{19}$ Previously, several strong and flexible multifunctional polymeric hydrogels with external stimuli sensitive materials ${ }^{17,19-24}$ have been developed; in these structures, the interstitial spaces between the polymer chains are filled with water, resulting in a potentially conductive medium for flexible sensors or electrolytes for flexible energy storage materials. ${ }^{1}$ In order to realize flexible energy storage devices, researchers have developed a double network hydrogel (DN hydrogel) electrolyte for supercapacitors with elasticity, reversibility and stretchability. ${ }^{25}$ Properly designed, the conductive hydrogel could even be applied under critical conditions. ${ }^{26}$ Yet, several pending issues still need to be resolved, such as avoiding the evaporation of water and reducing the high cost of the conductivity fillers to obtain highperforming polymeric hydrogels.

Ionic liquids (ILs) are low melting point salts that are often considered to be 'green' solvents for organic synthesis, catalysis, and electrochemistry. ${ }^{27-30}$ The electrochemical characteristics of ILs are very alluring, such as low volatility, thermal 
stability, high ionic conductivity, and a wide electrochemical operational window. ${ }^{17,22,31,32}$ These unique properties make ILs applicable as flexible and solid-state electrolytes. Ion gels derived from ILs are structurally similar to polymeric hydrogels, but they contain a large amount of ILs in the polymer network instead of water. In comparison to hydrogels, ILs have no solvent evaporation or leakage problems, but their conductivity is comparable to that of liquid electrolytes. ${ }^{33,34}$ Typically, ion gels can be classified into two categories based on whether ILs are involved in the polymerization reaction: polymerizing IL monomers and locking ILs in a polymer matrix. ${ }^{22}$ Due to restricted ion transport after polymerization, the conductivity of polymerized ion gels is often two orders lower than that of neat ILs. ${ }^{35-37}$ An alternative method for producing an ion gel is simply blending ILs and polymers, e.g., PVA or polyethylene oxide (PEO). ${ }^{21,38}$ Due to the weak mechanical properties of these ion gels, their applications in solid-state electrolytes and flexible sensors are limited. ${ }^{39}$ The ILs used for fabrication of ion gels are also often toxic and expensive, ${ }^{17,21,22}$ and may harm the human body when used as wearable devices. Therefore, more economical and nontoxic ion gel materials are desired when targeting the development of wearable and flexible devices.

Deep eutectic solvents (DESs) are a relatively new class of green solvents that are often considered to be a sub-class of ionic liquids. They can easily be produced by simply mixing two inexpensive commercially available raw materials at a specific temperature. ${ }^{40,41}$ The resulting mixture is self-associated through hydrogen bonding, often at a temperature below $100{ }^{\circ} \mathrm{C} .{ }^{42,43}$ Similar to ILs, DESs also show negligible vapor pressure, thermal and chemical stability, a wide electrochemical operational window, and high ionic conductivity. ${ }^{40,43}$ In comparison to ILs, DESs also offer several advantages, such as low cost, easy synthesis, green origin, and biodegradability. ${ }^{44}$ DESs have been widely used as electrolytes to produce nanoparticles electrochemically, especially the DES based on choline chloride (ChCl):urea (U) and choline chloride:ethylene glycol (EG). ${ }^{45}$ A DES prepared from choline chloride and orcinol was used for self-polymerization of 2-hydroxyethyl methacrylate (HEMA) to fabricate a highly transparent stretchable gel ${ }^{46}$ with good capacitive $\left(>200 \mathrm{~F} \mathrm{~g}^{-1}\right.$ ) behavior. Moreover, a DES prepared from choline chloride and glycerol was found to be a candidate with high potential for use as a non-aqueous liquid electrolyte in electrochemistry. ${ }^{47,48}$ Therefore, DESs can potentially be locked as a conductive medium in a polymer matrix without losing conductivity in order to construct a solid-state ion gel.

Apart from the above-mentioned applications of DESs, some studies have reported on a variety of DES applications in selective dissolution and extraction of biomaterials, such as chitin, ${ }^{44,49-51}$ cellulose, ${ }^{52-54}$ and lignin. ${ }^{54-56}$ Application of a DES in free-radical polymerizations for the synthesis of functional materials has also been described. ${ }^{43}$ From this perspective, the present work used a DES composed of choline chloride, urea, and glycerol (ChCl-U-G) as a medium for the free-radical polymerization of acrylamide (AM). Cellulose pulp from eucalyptus was directly dispersed in the DES to reinforce the mechanical property of the obtained ion gel. We successfully locked the conductive DES in the double network (cellulose and polyacrylamide) ion gel. The obtained ion gel is sensitive to external force, and it could be used as an electronic skin device to monitor grasping motions. This type of ion gel can also be used as a solid flexible electrolyte and as a separator for supercapacitors. Therefore, the ion gel was used to fabricate a symmetric supercapacitor. Moreover, the ion gel had a wide operating voltage window of $2 \mathrm{~V}$ due to the electrochemical stability of the DES. ${ }^{57}$

\section{Experimental}

\section{Materials}

Choline chloride (ChCl), urea, glycerol, and ammonium peroxydisulfate (APS) were purchased from Sinopharm Chemical Reagent Co., Ltd (Shanghai, China). AM and $N, N^{\prime}$-methylenebisacrylamide (MBA) were obtained from Macklin (Shanghai, China). All chemicals were analytical-reagent grade and used without any further purification. Commercial kraft cellulose pulp from eucalyptus was used as the dry sheets. It was torn into pieces by hand and dried for further use.

\section{Preparation of the DES}

The DES composed of ChCl-U-G, with a mole ratio of $1: 2:$ (0 to 0.9), was prepared in an Erlenmeyer flask and transferred to an oil bath with magnetic stirring at $100{ }^{\circ} \mathrm{C}$ until it became transparent. The resulting mixtures were categorized according to the mole ratio of glycerol as follows: $\mathrm{DES}_{0}, \mathrm{DES}_{0.125}$, $\mathrm{DES}_{0.25}, \mathrm{DES}_{0.5}, \mathrm{DES}_{0.7}$, and $\mathrm{DES}_{0.9}$. The electroconductivity (EC) of the DES was measured using an EC meter (FE30 Plus, MettlerToledo, Switzerland) at $25{ }^{\circ} \mathrm{C}$.

\section{Preparation of ion gel}

The conductive ion gel was prepared by in situ free radical polymerization of AM in the DES in the presence of cellulose pulp as a reinforcement material. In detail (shown in Table 1), first, APS was dissolved in the DES with a mass ratio of $3: 100$ to guarantee its dissolution and trigger the polymerization. Cellulose $(0,1,2,3$, and $4 \mathrm{wt} \%$ with respect to the weight of AM) was then dispersed in a portion of pure DES $(18 \mathrm{~g})$ at $100{ }^{\circ} \mathrm{C}$ until it formed a homogeneous solution, after which the mixture was cooled to room temperature. Next, $3 \mathrm{~g}$ of AM and $0.03 \mathrm{~g}$ of MBA were added to the DES, and the resultant mixture was stirred for $30 \mathrm{~min}$ at $40{ }^{\circ} \mathrm{C}$. Subsequently, the mixture was cooled to $25{ }^{\circ} \mathrm{C}$ and $2.06 \mathrm{~g}$ of APS-DES solution was added to the system with stirring for $5 \mathrm{~min}$. Then, the mixture was placed in

Table 1 Chemical compositions of the synthesized ion gels based on the DES

\begin{tabular}{llllll}
\hline Sample & AM $(\mathrm{g})$ & Cellulose $(\mathrm{wt} \%)$ & MBA $(\mathrm{g})$ & APS $(\mathrm{g})$ & DES $(\mathrm{g})$ \\
\hline DP0 & 3 & 0 & 0.03 & 0.06 & 20 \\
DP1 & 3 & 1 & 0.03 & 0.06 & 20 \\
DP2 & 3 & 2 & 0.03 & 0.06 & 20 \\
DP3 & 3 & 3 & 0.03 & 0.06 & 20 \\
DP4 & 3 & 4 & 0.03 & 0.06 & 20
\end{tabular}


a desiccator under a vacuum condition to remove any bubbles. Finally, the mixed solution was cast into moulds and sealed at $100{ }^{\circ} \mathrm{C}$ for $2 \mathrm{~h}$. The obtained ion gel was removed for further polymerization at room temperature for another $24 \mathrm{~h}$.

\section{Characterization of the ion gel samples}

DES was removed from the ion gel samples by impregnating the samples repeatedly in water. The hydrogels were lyophilized to obtain dry aerogels for further analysis (Fourier transform infrared [FTIR] spectroscopy, X-ray diffraction [XRD], and field emission scanning electron microscopy [FE-SEM]). FTIR spectra were recorded in the attenuated total reflectance (ATR) mode with a VERTEX $80 \mathrm{~V}$ spectrometer (Bruker, Rheinstetten, Germany) at room temperature in the range of $4000-400 \mathrm{~cm}^{-1}$ ( $4 \mathrm{~cm}^{-1}$ resolution). The crystalline structure of all the samples was characterized using a Bruker D8 ADVANCE instrument with $\mathrm{CuK} \alpha$ radiation $(\lambda=1.5406)(40 \mathrm{kV}, 30 \mathrm{~mA})$. The samples were recorded from a $2 \theta$ angle of $5^{\circ}$ to $55^{\circ}$ with a scanning speed of $4^{\circ}$ per minute. For morphology analysis, the samples were coated with a thin gold (Au) layer under a vacuum condition using a sputter coater and imaged by FE-SEM with a voltage of $20.0 \mathrm{kV}$ (Hitachi SU8220, Tokyo, Japan).

All the mechanical properties were measured at room temperature using a universal mechanical testing machine (5966, INSTRON, Canton, MA, USA) at a crosshead speed of $50 \mathrm{~mm}$ $\min ^{-1}$. For the tensile stress-strain test, the samples were shaped in $50 \mathrm{~mm} \times 10 \mathrm{~mm} \times 5 \mathrm{~mm}$ polytetrafluoroethylene (PTFE) molds. The unconfined compression stress $(\sigma)$-strain $(\varepsilon)$ tests were performed on cylindrical samples $(20 \mathrm{~mm}$ in diameter and $20 \mathrm{~mm}$ in height). The self-recovery properties were investigated with a compressive loading-unloading test using 50 time loops.

The ion gel samples, with a size of $50 \mathrm{~mm} \times 5 \mathrm{~mm} \times 5 \mathrm{~mm}$, were sandwiched between two ends. The resistance of the ion gels was then determined using linear sweep voltammetry (LSV) on an electrochemical workstation (CHI660E, Chenhua Instrument Inc., Shanghai, China), and it was calculated using Ohm's law (eqn (1)):

$$
R=U / I
$$

where $R$ is the resistance $(\Omega), U$ is the open circuit potential of the conductive ion gel $(\mathrm{V})$, and $I$ is the current corresponding to the open circuit potential (A). The conductivity of the ion gel was calculated by eqn (2):

$$
\sigma=\frac{1}{R} \frac{L}{S}
$$

where $\sigma, L$, and $S$ are the conductivity $\left(\mathrm{S} \mathrm{cm}^{-1}\right)$, thickness $(\mathrm{cm})$, and electrode area $\left(\mathrm{cm}^{2}\right)$, respectively.

The real-time electrical signals of the ion gel sensor were recorded on an electrochemical workstation (CHI660E, China) using the amperometric $i-t$ curve program under a constant voltage of $5.0 \mathrm{~V}$. The ion gel sample, with dimensions of $40 \mathrm{~mm}$ $\times 5 \mathrm{~mm} \times 5 \mathrm{~mm}$, was attached to the joint of the back side of the index finger to monitor the strain-induced changes in the electrical current. The relative change of the resistance
$\left(\Delta R / R_{0}, \Delta R\right.$ is the resistance change and $R_{0}$ is the initial resistance) was calculated using Ohm's law.

Porous carbon (PC), with a specific surface area of $1573 \mathrm{~m}^{2} \mathrm{~g}^{-1}$ and prepared from waste palm, was used as an active material for the supercapacitor. The PC, acetylene black, and PTFE (mass ratio, 8:1:1) were mixed in ethanol and ground in an agate mortar to obtain a black slurry. The slurry was evenly spread on a slice of nickel foam, and then dried in a vacuum oven at $100{ }^{\circ} \mathrm{C}$ for $8 \mathrm{~h}$. The electrodes were then pressed under $10 \mathrm{MPa}$ for $1 \mathrm{~min}$ with a loading of $2-3 \mathrm{mg} \mathrm{cm} \mathrm{cm}^{-2}$. The symmetric supercapacitors were assembled from two identical electrodes. The ion gel was directly used as the electrolyte and separator to prepare the symmetric electric double layer supercapacitor (EDLC). The electrochemical properties of the EDLS were investigated by using an electrochemical workstation (Reference 600+, Gamry). Cyclic voltammetry (CV) measurements were carried out at different scan rates $(5,10,20,50$, and $100 \mathrm{mV} \mathrm{s}^{-1}$ ) in the potential window ranging from $0 \mathrm{~V}$ to 2 V. Galvanostatic charge/discharge (GCD) was recorded at different current loads of $0.2,0.3,0.4,0.5$, and $1.0 \mathrm{~A} \mathrm{~g}^{-1}$. The electrochemical impedance spectroscopy (EIS) tests were operated at $5 \mathrm{mV}$ amplitude, and the frequency ranged from $0.01 \mathrm{~Hz}$ to $100 \mathrm{kHz}$ at the open circuit potential.

The single electrode-specific capacitance, energy density, and power density were determined using eqn (3)-(5):

$$
\begin{gathered}
C_{\mathrm{s}}=\frac{4 \cdot I \cdot \Delta t}{m \cdot \Delta V} \\
E=\frac{C_{\mathrm{s}} \cdot \Delta V^{2}}{2 \cdot 4 \cdot 3.6} \\
P=E \cdot t^{-1}
\end{gathered}
$$

where $C_{\mathrm{s}}$ denotes the specific capacitance $\left(\mathrm{F} \mathrm{g}^{-1}\right), I$ is the discharge current (A), $\Delta t$ is the discharge time (s), $\Delta V$ is the voltage change $(\mathrm{V})$, and $m$ is the total mass of the loaded PC in each electrode (g). $E$ is the energy density $\left(\mathrm{W} \mathrm{h} \mathrm{kg}^{-1}\right)$ and $P$ is the power density $\left(\mathrm{kW} \mathrm{kg}^{-1}\right)$.

\section{Results and discussion}

\section{Design and synthesis of ion gel in DES}

The present study used a DES that was composed mainly of $\mathrm{ChCl}$ and urea, which are non-toxic, low-cost compounds that possess excellent dispersibility of cellulose. However, the ionic conductivity of this binary DES is low. Therefore, a small amount of glycerol was added to increase its ionic conductivity. Fig. 1 shows the effect of glycerol on the ionic conductivity of the ChCl-urea DES. Obviously, the addition of glycerol dramatically increased the conductivity of ChCl-urea. The conductivity of ChCl-U-G with a molar ratio of $1: 2: 0.125$ was $0.822 \mathrm{mS} \mathrm{cm}^{-1}$, which is comparable to that of the $\mathrm{ChCl}-$ glycerol DES ${ }^{58}\left(0.106-1.047 \mathrm{mS} \mathrm{cm}^{-1}\right.$ at $\left.20{ }^{\circ} \mathrm{C}\right)$. Further addition of glycerol does not affect the conductivity too much. Taking into account the possible leakage of additional glycerol from the DES after the polymerization of AM and its possible negative 


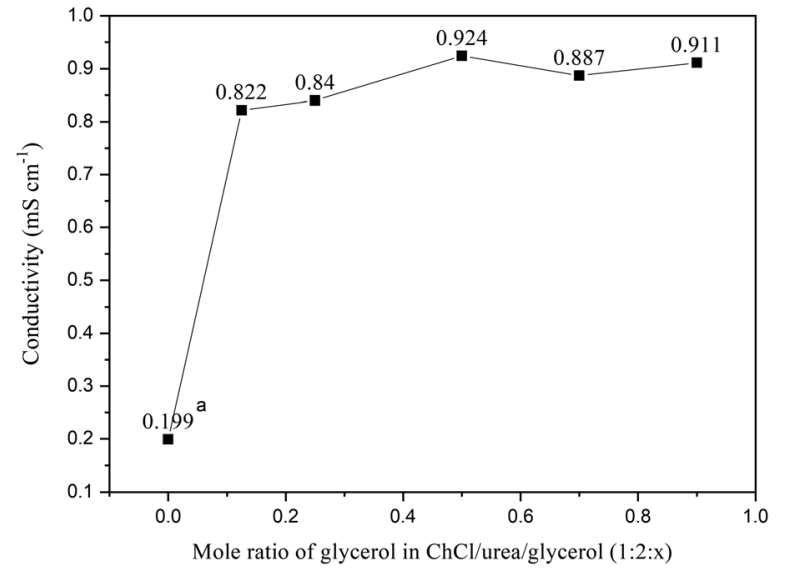

Fig. 1 Ionic conductivity of $\mathrm{ChCl}-\mathrm{U}-\mathrm{G}$ based DESs with different amounts of glycerol at $25^{\circ} \mathrm{C}$ (a. the conductivity of $\mathrm{ChCl}$-urea at $40{ }^{\circ} \mathrm{C}^{61}$ ).

influence on the polymerization process, ChCl-U-G with a molar ratio of 1:2:0.125 was selected as the solvent for dispersion of cellulose and the medium for the polymerization of polyacrylamide (PAAM) to synthesize the conductive ion gel.

PAAM-based hydrogels are non-toxic and biologically inert, which give them the potential to be applied in drug delivery, wound dressing, and smart devices. ${ }^{59,60}$ However, a pure PAAM hydrogel synthesized in water is brittle, and it easily fractures upon stretching, which limits its application in flexible and wearable devices. Therefore, efforts have been made to improve the mechanical properties of PAAM hydrogels using materials, such as nanofillers. ${ }^{62-64}$ Nanocellulose is widely used in this area due to its abundance in nature, its biocompatibility, and its availability. In the present work, a facile one-pot fabrication of PAAM containing a DES ion gel is shown in Fig. 2a. Cellulose fibers were directly used to reinforce the mechanical properties of the ion gel. This straightforward and simple approach does not require the use of cellulose nanomaterials. ${ }^{64}$ The ion gel without any cellulose (DP0) was highly transparent, and the transparency decreased as the content of cellulose increased (Fig. 2b). The obtained ion gel showed strong adhesion towards rubber and stainless steel (Fig. 2c). Interestingly, the obtained ion gel was highly stretchable, and it showed excellent mechanical properties in comparison to the ion gel obtained in water (without DES) $)^{59}$ (Fig. 2d). Moreover, the locked DES in the polymer crosslinked network provided electrical conductivity for the ion gel and extended its potential for use in different electronic applications.

\section{Mechanical and conductivity properties of the ion gels}

As shown in Fig. 3a, the contribution of cellulose to the mechanical properties of the ion gel by changing the cellulose/ DES mass ratio was evaluated first. The resulting ion gels were categorized based on the amount of cellulose as follows: DP0, DP1, DP2, DP3, and DP4 (Table 1). All the ion gel samples were highly stretchable, having a strain at break $>200 \%$. However, cellulose played a critical role in the mechanical properties of the ion gels, and the tensile strength of the ion gels increased with increasing cellulose content, especially in the case of DP3 and DP4, which had a tensile strength of $45.4 \mathrm{kPa}$ and $55.5 \mathrm{kPa}$, respectively. In comparison to the pure ion gel, the tensile strength of the cellulose-reinforced ion gels improved by 3.2-3.9-fold. The elongation of the ion gels increased with the addition of cellulose to the samples that contained a lower amount of cellulose, but it decreased as the concentration of cellulose increased. The role of cellulose is likely ascribed to its ability to transfer the external load, similar to that in a wood structure. ${ }^{64}$ However, its rigidity may cause the low elongation of ion gels. This phenomenon is similar to what was previously reported with poly(acrylic acid) hydrogel reinforced with cellulose nanocrystals. ${ }^{65}$ Moreover, the excellent mechanical properties are attributed to the trapped DES, which formed a large number of hydrogen bonds between the PAAM chains. Thus, the DES plays a dual role as a filler in a polymer matrix and as a conductive agent for ion gels. The tensile strength of the DES-based double network ion gel is relatively lower than that of the more recently reported ion gel, ${ }^{66-69}$ in which the tensile strengths of the obtained ion gels were all above $100 \mathrm{kPa}$, even up to tens of megapascals. But, when used as a solid state electrolyte, the compressive property is more important.

The conductivity of ion gels was calculated according to eqn (1) and (2) by testing their resistance using the LSV program conducted on the electrochemical workstation. It was noted that the addition of cellulose to DES/PAAM had a relatively (a)

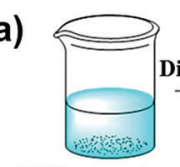

ChCl-urea-glycerol

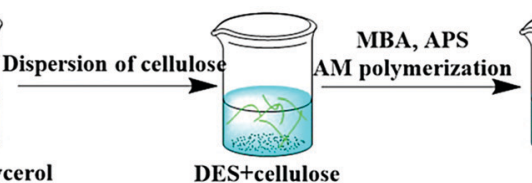

DES+cellulose

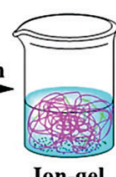

Ion-gel (c)

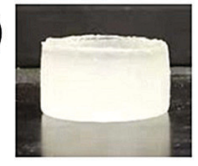

(d)

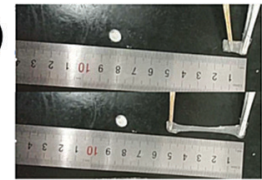

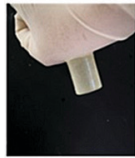
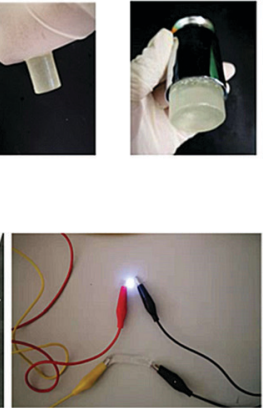

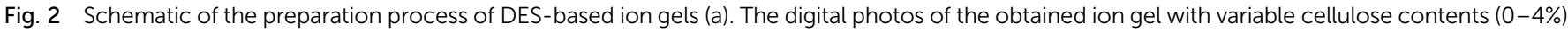

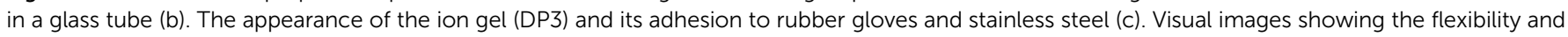
conductivity of the ion gel (DP3) (d). 

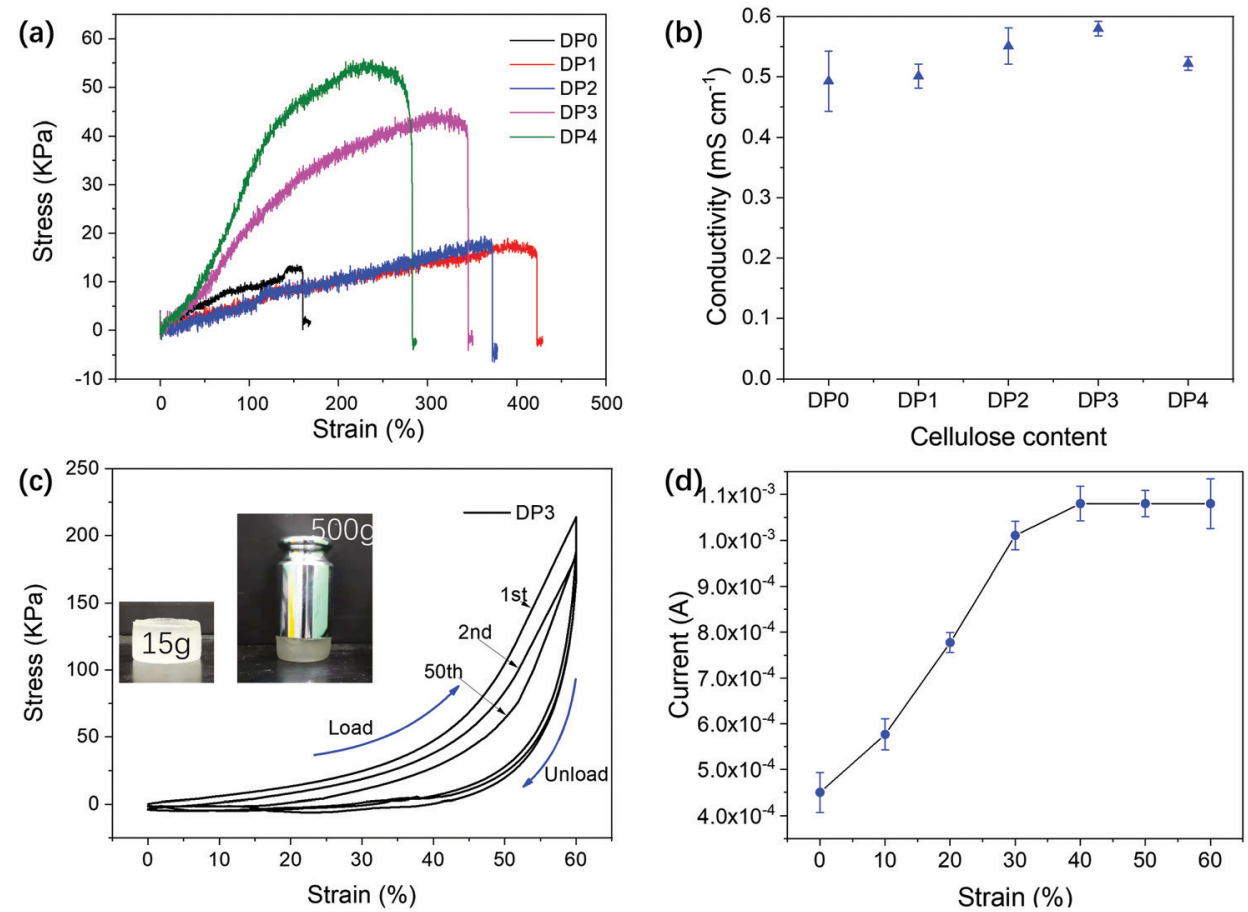

Fig. 3 Tensile strength (a) and conductivity (b) of the ion gels with different cellulose contents (0-4\%). Typical compressive stress-strain cycles of DP3, insets are cylindrical ion gel and a weight on ion gel (c) and current change of DP3 under various strains (d).

small impact on the conductivity of the ion gel (Fig. 3b). Actually, DP3 showed the highest conductivity among the ion gels, which was likely due to the extra ion diffusion pathway realized through the electrolyte uptake by the cellulose fibers. ${ }^{70}$ PEO incorporating various ILs is considered as a prevalent approach to make solid state electrolytes. The ionic conductivity of the obtained ion gel ranges from 0.01 to $2 \mathrm{mS} \mathrm{cm}^{-1} \cdot{ }^{38,71}$ Also, one study reported the superior ionic conductivity property of PVA/IL ion gel. ${ }^{72}$ In contrast to IL-based ion gels, as designer solvents, DES-based ion gels are a promising alternative for making solid state electrolytes. Consequently, due to the mechanical characteristics and conductivity of the ion gel samples, DP3 was used as the representative sample for further analysis.

It is well known that pure PAAM hydrogels show a ductile fracture with strain under a compression test. ${ }^{64}$ However, in the present study, the cellulose-reinforced DES ion gels showed tough and highly resilient features (Video S1, ESI $\dagger$ ). The cyclic strain-stress curves of DP3 at a fixed strain of $60 \%$ are shown in Fig. 3c. The hysteresis loop is obvious because of the strong ion gel, which offers remarkable energy dissipation from bond breakage. $^{73}$ The cylindrical ion gel was able to withstand a weight as high as $500 \mathrm{~g}$ (Fig. 3c, inset). It could be recovered rapidly after the release of compressive force or even compaction by rolling without any cracks (Video S2, ESI $\dagger$ ). These superior properties may be attributed to the unique structure of the network, which consists of intensive hydrogen bonds between the PAAM chains and DES. When the DP3 ion gel was applied as a solid-state electrolyte for EDLCs, the compressive force had a positive effect on its conductivity, as depicted in Fig. 3d. The current of the ion gel first increased; it was then constant as the compressive strain increased. The current of DP3 was only $4.5 \times 10^{-4} \mathrm{~A}$ under $0 \%$ compression, and it quickly increased to $10.8 \times 10^{-4}$ A under $40 \%$ compression, which is 2.4 times higher than the initial value. This result also indicated that the conductivity of the ion gel could be increased by more than 1.44 times according to eqn (2), and by assuming that the area of the ion gel is constant (the real area of an ion gel expands under compressive force). This can be ascribed to the fact that compressive force breaks the non-covalent bonds and weakens the storage modulus, which increases the mobility of the ions in the gel. ${ }^{21}$ Therefore, DES ion gels can be considered to be excellent candidates for solid-state electrolytes in supercapacitors.

\section{Physicochemical properties of the ion gel}

To better understand the interactions between the cellulose reinforcement and PAAM, FTIR and XRD analyses of the ion gels were performed. After exchanging the DES of the ion gel with water, no cellulose fiber was observed in the water solution, which indicates that the cellulose was well bonded and embedded in the PAAM matrix. The obtained hydrogel (without DES) was fragile, which further demonstrated the important role of DES in improving the mechanical properties of ion gels. The FTIR spectra of the cellulose and the DP0 and DP3 aerogels are shown in Fig. 4a. The intense absorption peak at $3332 \mathrm{~cm}^{-1}$ is due to the stretching vibration of $\mathrm{O}-\mathrm{H}$. The other characteristic absorption peaks at $1023 \mathrm{~cm}^{-1}$ and $1159 \mathrm{~cm}^{-1}$ are due to $\mathrm{C}-\mathrm{OH}$ stretching and the asymmetric stretching vibration of $\mathrm{C}-\mathrm{O}-\mathrm{C}$ for cellulose. For DP0, the broad bands around $3186 \mathrm{~cm}^{-1}$ and $3338 \mathrm{~cm}^{-1}$ were due to the stretching 

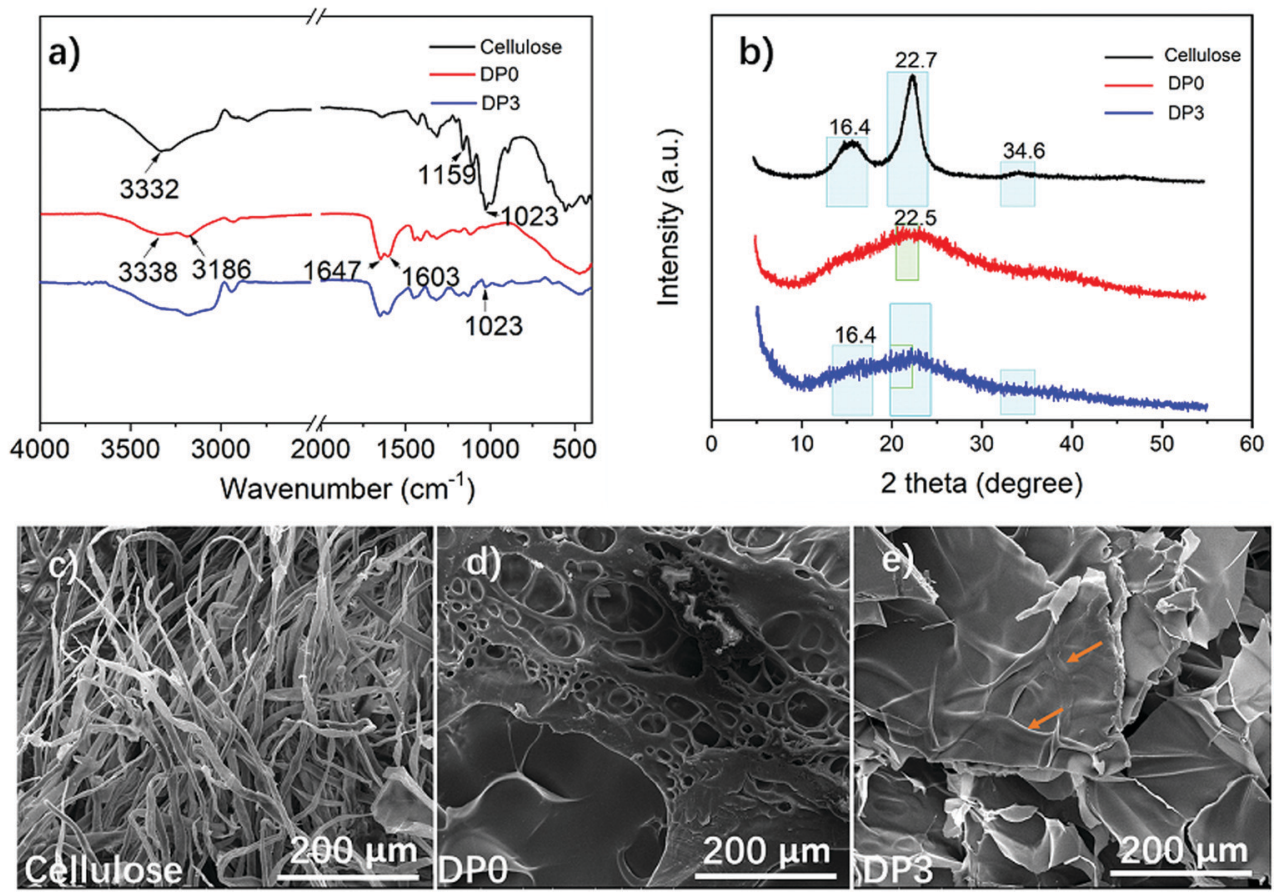

Fig. 4 FTIR spectra (a), XRD patterns (b) and FESEM images of cellulose (c), DPO (aerogel, d) and DP3 (aerogel, e).

vibration of $\mathrm{N}-\mathrm{H}$. The typical peaks at $1647 \mathrm{~cm}^{-1}$ and $1603 \mathrm{~cm}^{-1}$ were attributed to the carbonyl stretching (amide I) vibration and the $\mathrm{N}-\mathrm{H}$ bending vibration (amide II). ${ }^{74}$ The DP3 spectra showed peaks that were similar to the peaks of the DP0 spectra; however, the intensity of the peak at $1023 \mathrm{~cm}^{-1}$ increased, which is due to the $\mathrm{C}-\mathrm{OH}$ stretching from cellulose. Fig. $4 \mathrm{~b}$ shows the XRD patterns of the cellulose and the DP0 and DP3 aerogels. The main peaks around $16.4^{\circ}, 22.7^{\circ}$, and $34.6^{\circ}$ are typical peaks for cellulose. ${ }^{75}$ DP0 showed a broad peak at $22.5^{\circ}$, which is a characteristic peak of PAAM. ${ }^{76}$ As seen for the DP3 hybrid aerogel, all of these corresponding peaks confirm that cellulose was successfully embedded in the PAAM matrix. The morphology of the cellulose and the DP0 and DP3 aerogels is shown in Fig. $4 \mathrm{c}-\mathrm{e}$. The diameter of the cellulose fibers was about $10 \mu \mathrm{m}$. The morphology of the DP0 aerogel exhibited a porous structure, which is similar to that of the ion gel polymerized under a water condition. ${ }^{64}$ For the DP3 aerogel, the cellulose fibers were clearly visible and embedded in the structure (as noted by arrows). Therefore, inter- and/or intra-molecular interactions existed between the PAAM and cellulose. The formation of the hydrogen bonds or van der Waals interactions between these two polymers is beneficial for improving the mechanical properties of ion gels.

\section{Applied as strain sensor}

To illustrate the conductivity of the ion gel, a battery powered circuit containing an LED bulb was used. The lightened bulb connected through the DP3 ion gel is shown in Fig. 5a. The luminance was very stable with various deformations of the DP3 ion gel, which indicates its good conductivity. The LED bulb was only extinguished when the ion gel was cut and separated to form an open circuit. Interestingly, the LED bulb recovered and showed the same luminance as the primary ion gel when the cracked ion gels were only put in contact through a small surface. The luminance of the two pasted surfaces was also the same as that of the primary and touched surface ion gels. Most of the reported conductive hydrogels are not as sensitive when reconnecting two cut surfaces, but they may recover their conductivity by self-healing after several minutes or hours to regain ion diffusion equilibrium..$^{20,77,78}$ The obtained results demonstrated the excellent conductivity, repeatability, and quick restoration of the electrical performance of DES-based ion gels. This performance is likely due to the porous structure of PAAM, which entraps highly conductive DES and enables the formation of an even and continuous ion gel. The ion gel also exhibits mechanosensitive properties. The luminance of the LED gradually decreased with increasing tensile strain, as shown in Video S3 (ESI $\dagger$ ). Therefore, the DES-based ion gel has the potential for use in applications such as electronic skin or smart devices.

The strain-sensitive ion gel was applied to monitor joint motion by fixing it onto the joint of a finger. Due to the different tensile deformations of the ion gel during the grasping motion (Fig. 5b), its resistance could be altered. Fig. 5c shows the corresponding current change of the grasping motion. With the step-by-step changes in the grasping motion and tensile deformation, the increased resistance of the ion gel varied systematically. After the palm was opened, the stretched ion gel became completely relaxed and the resistance could be restored to its initial value. A continuous bending test was used to illustrate the rapid response, repeatability, and stability of the sensor (Fig. 5d and e).

In summary, these results demonstrate that the DES-based ion gel exhibits excellent real-time monitoring ability and 

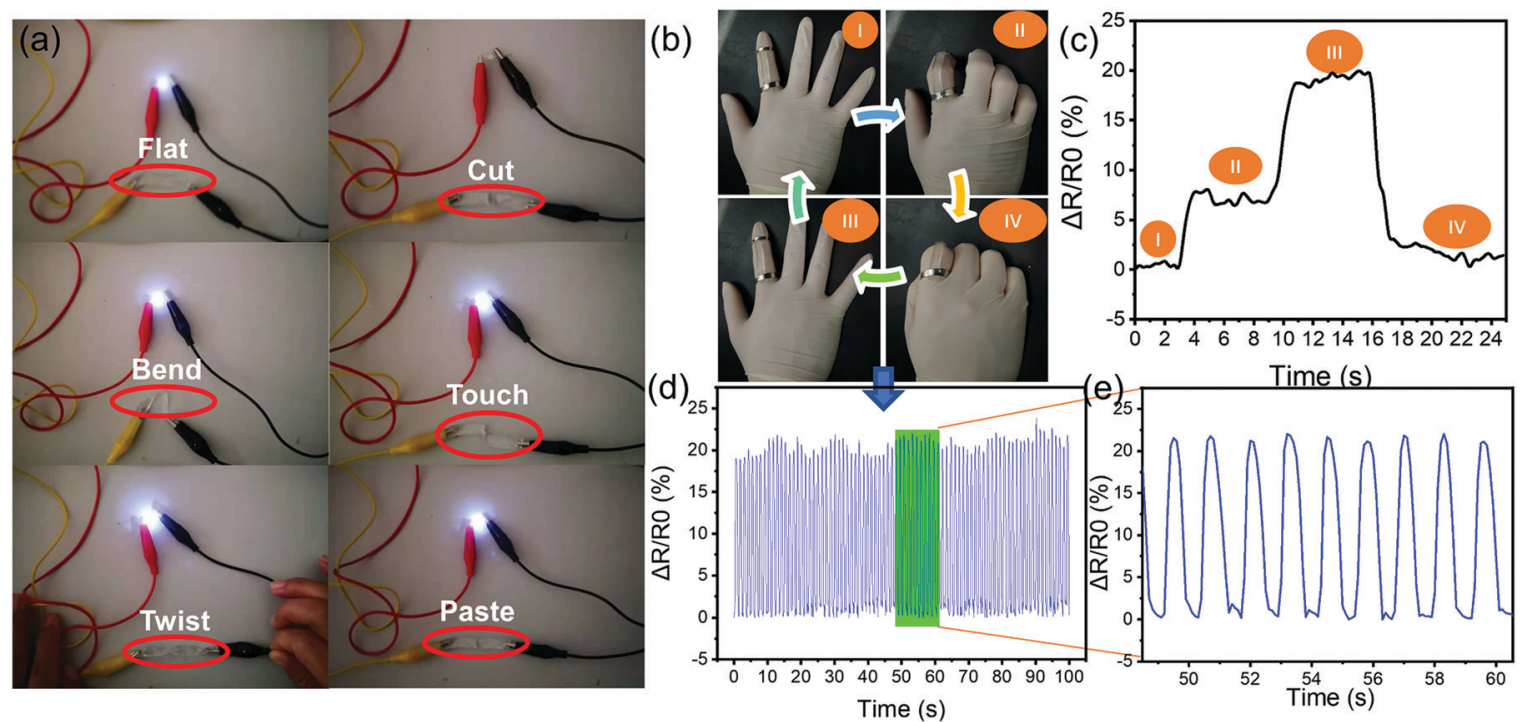

Fig. 5 Photographs demonstrating the electrical conductivity of the ion gel with various deformations (a). The ion gel as electronic skin to monitor the grasping motion (b) and its corresponding current change (c). Relative resistance changes of the ion gel as a wearable sensor in monitoring finger bending motion ( $d$ and $e$ ).

possesses the strain-sensitive properties of conductive elastomers. It is important to note that all of the chemicals used in the present study are non-volatile and have a higher boiling point than water, which enabled the ion gels to work in a wide temperature range. More importantly, the polymer matrix (PAAM) used in this study is bio-safe, ${ }^{79}$ and the electrically-conductive mixture (DES) is low-cost and nontoxic, while the reinforcement (cellulose) is a natural and readily available biomaterial. Therefore, the DES-based ion gel can be considered to be a cost-effective, accessible, and competitive candidate for fast responsive actuators and sensory skin in robotic systems.

\section{Ion gel as a solid-state electrolyte for symmetric supercapacitors}

The ion gel (DP3) film was prepared by casting it in a glass Petri dish and curing it. The thickness of the film was about $0.5 \mathrm{~mm}$. The ion gel film was directly used as a separator and solid-state electrolyte for fabrication of a coin cell type EDLC. The electrochemical performance of the supercapacitor was investigated in a two-electrode configuration. Fig. 6a shows the cyclic voltammograms at scan rates ranging from $5 \mathrm{mV} \mathrm{s}^{-1}$ to $100 \mathrm{mV} \mathrm{s}^{-1}$. Due to its good mechanical properties, the ion gel film successfully separated the positive and negative electrodes of the EDLC because no short-circuit was observed. The shape of the CV curves is almost quasi-rectangular at a lower scan rate, which indicates that two capacitive layers are formed at the electrodeelectrolyte interface. ${ }^{80}$ When the scan rate increased to $50 \mathrm{mV} \mathrm{s}^{-1}$, the shape significantly changed to a shuttle form. This deviation is associated with the high internal resistance in the system. The GCD tests of the EDLC at different current densities are shown in Fig. 6b. The GCD curves are symmetrical triangles, and the relationship between voltage and time is linear under constant current charging-discharging; thus, the interface between the electrode and electrolyte only underwent reversible ion adsorption and desorption, but no faradaic reaction.

The resistance of the proposed ion gel still requires improvement. The specific capacitances of the EDLC, calculated from the GCD results, were $161.8,141.8,132.1,125.6$, and $76.6 \mathrm{~F} \mathrm{~g}^{-1}$ at a current density of $0.2,0.3,0.4,0.5$, and $1.0 \mathrm{~A} \mathrm{~g}^{-1}$, respectively. This result is comparable to PEO and 1-ethyl-3methylimidazolium bis(trifluoromethyl sulfonyl)-imide (EMImTFSI)based ionic liquid gel electrolytes and activated carbon-based electrodes at a current density of $1.0 \mathrm{~A} \mathrm{~g}^{-1}$ (ranging from $12.04 \mathrm{~F} \mathrm{~g}^{-1}$ to $\left.90.28 \mathrm{~F} \mathrm{~g}^{-1}\right){ }^{81}$ The specific capacitances decreased as the current density increased, which further proves the high internal resistance in the system.

The Nyquist impedance spectrum of the EDLC obtained from the EIS test is shown in Fig. 6c. The Nyquist plot showed a semicircle behavior at high frequencies, which is associated with the electron-transfer reaction at the electrolyte/electrode interface. That was followed by a straight line, which is almost parallel to the imaginary axis of the impedance in the low frequency region corresponding to the Warburg impedance associated with the charge diffusion process in the electrode materials. ${ }^{22}$ This system showed a typical behavior of capacitive and porous interfaces.

The intercept on the real axis at high frequencies represents the internal resistance $\left(R_{\mathrm{i}}, 58.1 \Omega\right)$, i.e., the resistance of the electrode, connection resistance, and electrolyte of the device. The diameter of the semicircle is related to the charge transfer resistance $\left(R_{\mathrm{ct}}, 37.2 \Omega\right)$. As expected, $R_{\mathrm{i}}$ is very high due to the high resistance of the ion gel electrolyte. The charge transfer resistance is related to the electrode-electrolyte interface and the pore structure of the electrodes. The resistance arising from the electrodes is likely negligible because a previous study showed that activated carbon is a suitable material for 

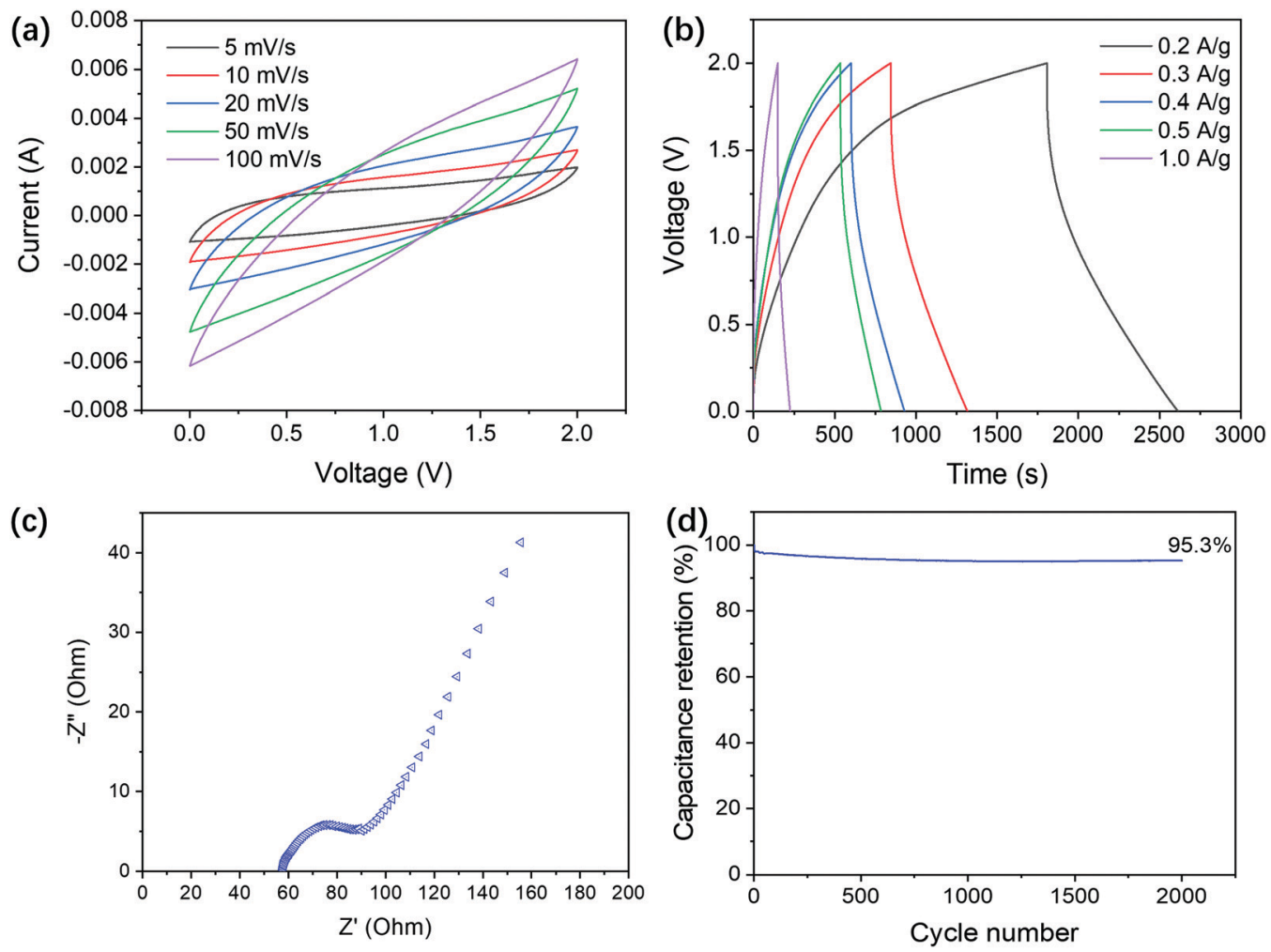

Fig. 6 Electrochemical properties of EDLC using DP3 as a solid-state electrolyte. CV curves measured at different scan rates (a), GCD profiles measured at different current densities (b), Nyquist plot (c) and cycling stability of the DP3 ion gel-based supercapacitor at a current density of $1.0 \mathrm{~A} \mathrm{~g}^{-1}$ (d).

supercapacitors. ${ }^{82}$ The main electronic resistance is derived from the interfacial resistance between the inhomogeneity of the electrolyte-electrode interfaces. The performance of the DES-based ion gel as an electrolyte for a supercapacitor is comparable to those of IL incorporated polymer electrolytes with a charge transfer resistance ranging from $30 \Omega$ to $60 \Omega .{ }^{80,83}$ The long-term cyclability of the DES-based ionic gel supercapacitor was investigated using the GCD method at a current density of $1.0 \mathrm{~A} \mathrm{~g}^{-1}$ for over 2000 cycles (Fig. $6 \mathrm{~d}$ ). The results showed that the proposed DES-based ionic gel supercapacitor had good durability with a remarkable capacitance retention of $95.3 \%$ after 2000 cycles.

Based on the GCD results, the relationships between energy and the power densities are portrayed in Ragone plots (Fig. 7a). The DES-based ion gel electrolyte supercapacitor showed a high energy density of $22.47 \mathrm{~W} \mathrm{~h} \mathrm{~kg}^{-1}$ at a power density of $0.11 \mathrm{~kW} \mathrm{~kg}^{-1}$ due to the wide operating voltage of $2.0 \mathrm{~V}$ and the excellent capacitance of the EDLC. The DES-based ion gel showed a relatively high internal resistance resulting from the decrease in the capacitance of the EDLC as the current density increased. The energy density decreased with the increased power density; this could be ascribed to the limitation of the ion mobility of the DES-based ion gel electrolyte during the fast chargedischarge cycle. However, the energy density $\left(10.64 \mathrm{~W} \mathrm{~h} \mathrm{~kg}^{-1}\right)$ is still acceptable at a power density of $0.5 \mathrm{~kW} \mathrm{~kg}^{-1}$. In comparison to different ionic liquid-based ion gel electrolytes for the EDLC system, both the energy and power densities are comparable (Fig. 7a). To demonstrate the practical application of
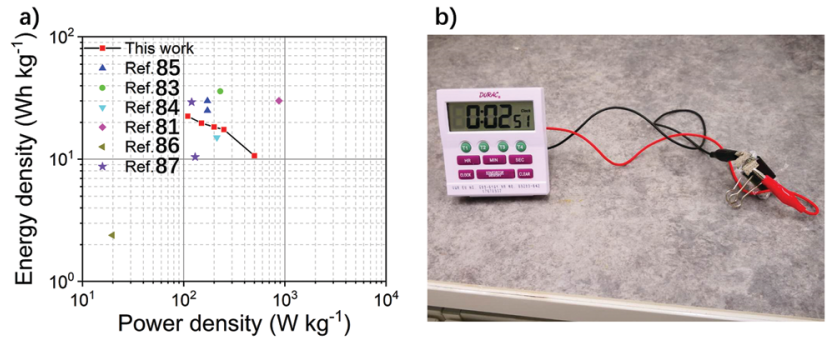

Fig. 7 Ragone plots of the EDLC with the DES-based ion gel electrolyte and some of the reported values of ionic liquid-based ion gel electrolytes (a), and the EDLC fabricated with the DP3 ion gel as the power source of a clock (b).

supercapacitors, two supercapacitors were connected in series (Fig. 7b) and used as a power source for a clock. Table 2 shows a comparison of the DES-based ion gel electrolyte with the ionic liquid-based ion gel electrolytes in the EDLC system. Although the conductivity of the DES-based ion gel is lower, it is compatible with the electrodes, and it has a wide operating voltage window, resulting in a high performance EDLC. These results indicate that the DES-based ion gel electrolyte is feasible as a low-cost, solid-state electrolyte. However, the conductivity of ion gels must be improved. Since DESs are tailorable and easy to make, the DES-based ion gel with a high ion conductivity could be produced and applied as a new type of competitive solid-state electrolyte for flexible and high-performance supercapacitors. 
Table 2 Comparison of the performance of the DP3-based ion gel with other IL electrolytes from the literature (the electrodes are all activated carbon)

\begin{tabular}{|c|c|c|c|c|}
\hline Gel polymer & $\sigma\left(\mathrm{mS} \mathrm{cm}^{-1}\right)$ & $C_{\mathrm{s}}\left(\mathrm{F} \mathrm{g}^{-1}\right) @ 1 \mathrm{~A} \mathrm{~g}^{-1}$ & Voltage (V) & Ref. \\
\hline PVDF-HFP/EMimTFSI + LiTFSI & 4.5 & 108 & 2.0 & 84 \\
\hline EMImTFSI-PEO-FPC & 6.7 & 83.39 & 3.5 & 81 \\
\hline $\mathrm{PVA} / \mathrm{CH}_{3} \mathrm{COONH}_{4} / \mathrm{BmImCl}$ & 7.31 & 31.28 & 1 & 86 \\
\hline PVA- $\mathrm{Li}_{2} \mathrm{SO}_{4}$-BMIMI & 46.0 & $\sim 350$ & 1.5 & 87 \\
\hline PVA-Li ${ }_{2} \mathrm{SO}_{4}-\mathrm{BMIMCl}$ & 37.2 & $\sim 130$ & 1.5 & 87 \\
\hline
\end{tabular}

\section{Conclusions}

This study proposed a new type of DES-based ion gel. The ion gel was produced by free radical polymerization of AM in a DES of ChCl-U-G. Cellulose pulp fibers were used as a reinforcement to increase the mechanical properties of the ion gel. DES was successfully locked in a PAAM polymer matrix through noncovalent bonding (hydrogen bond and van der Waals' interactions). The obtained ion gel was conductive, flexible, strong, and recoverable. The tensile strength and strain could be improved by 3.2-fold and 2.2-fold, respectively, by the addition of $3 \%$ cellulose. The ion gel even resisted the critical compression strain without any cracks. The sensor testing results indicated that the DES-based ion gel could be an excellent responsive actuator and sensory skin for robotic systems.

The DES-based ion gel also possessed high ionic conductivity $\left(0.58 \mathrm{mS} \mathrm{cm}^{-1}\right)$ and a wide operating voltage window $(2 \mathrm{~V})$. The performance of the EDLC system fabricated with this ion gel (solid-state electrolyte) was evaluated using CV and GCD cycling. The EDLC exhibited a high specific capacitance of $76.6 \mathrm{~F} \mathrm{~g}^{-1}$ at $1.0 \mathrm{~A} \mathrm{~g}^{-1}$ and a high energy density of $10.64 \mathrm{~W} \mathrm{~h} \mathrm{~kg}^{-1}$ at a power density of $500 \mathrm{~W} \mathrm{~kg}^{-1}$. It also exhibited excellent cyclic stability up to 2000 cycles at a current density of $1.0 \mathrm{~A} \mathrm{~g}^{-1}$, thereby achieving a high capacitance retention of $95.3 \%$. This study provided an application of DES in electronic materials. Finding a highly conductive and low expenditure DES-based ion gel could add much value and boost the development of both solidstate electrolytes for supercapacitors and other electronic materials.

\section{Conflicts of interest}

There are no conflicts to declare.

\section{Acknowledgements}

This research was supported by grants from the National Natural Science Foundation of China (31370567), the Doctorate Fellowship Foundation of Nanjing Forestry University, the National First-Class Disciplines, the Priority Academic Program Development of Jiangsu Higher Education Institutions, and the Academy of Finland project “Bionanochemicals” (298295).

\section{Notes and references}

1 Z. F. Wang, H. F. Li, Z. J. Tang, Z. X. Liu, Z. H. Ruan, L. T. Ma, Q. Yang, D. H. Wang and C. Y. Zhi, Adv. Funct. Mater., 2018, 28, 1804560.

2 Y. Huang, M. S. Zhu, Y. Huang, Z. X. Pei, H. F. Li, Z. F. Wang, Q. Xue and C. Y. Zhi, Adv. Mater., 2016, 28, 8344-8364.

3 W. Liu, M. S. Song, B. Kong and Y. Cui, Adv. Mater., 2017, 29, 1603436.

4 J. Lee, W. Kim and W. Kim, ACS Appl. Mater. Interfaces, 2014, 6, 13578-13586.

5 J. R. Miller and P. Simon, Science, 2008, 321, 651-652.

6 X. Peng, L. L. Peng, C. Z. Wu and Y. Xie, Chem. Soc. Rev., 2014, 43, 3303-3323.

7 Y. P. Fu, X. Cai, H. W. Wu, Z. B. Lv, S. C. Hou, M. Peng, X. Yu and D. C. Zou, Adv. Mater., 2012, 24, 5713-5718.

8 P. P. Li, Z. Y. Jin, L. L. Peng, F. Zhao, D. Xiao, Y. Jin and G. H. Yu, Adv. Mater., 2018, 30, 1800124.

9 K. Wang, P. Zhao, X. M. Zhou, H. P. Wu and Z. X. Wei, J. Mater. Chem., 2011, 21, 16373-16378.

10 J. Liu, M. Hu, J. Wang, N. Nie, Y. Wang, Y. Wang, J. Zhang and Y. Huang, Nano Energy, 2019, 58, 338-346.

11 C. J. Yu, C. Masarapu, J. P. Rong, B. Q. Wei and H. Q. Jiang, Adv. Mater., 2009, 21, 4793-4797.

12 X. Li, T. L. Gu and B. Q. Wei, Nano Lett., 2012, 12, 6366-6371.

13 X. L. Chen, L. B. Qiu, J. Ren, G. Z. Guan, H. J. Lin, Z. T. Zhang, P. N. Chen, Y. G. Wang and H. S. Peng, Adv. Mater., 2013, 25, 6436-6441.

14 C. Zhao, C. Y. Wang, Z. L. Yue, K. W. Shu and G. G. Wallace, ACS Appl. Mater. Interfaces, 2013, 5, 9008-9014.

15 Y. Z. Xie, Y. Liu, Y. D. Zhao, Y. H. Tsang, S. P. Lau, H. T. Huang and Y. Chai, J. Mater. Chem. A, 2014, 2, 9142-9149.

16 P. M. Xulu, G. Filipcsei and M. Zrínyi, Macromolecules, 2000, 33, 1716-1719.

17 E. Kamio, T. Yasui, Y. Iida, J. P. Gong and H. Matsuyama, Adv. Mater., 2017, 29, 1704118.

18 C. X. Ma, W. Lu, X. X. Yang, J. He, X. X. Le, L. Wang, J. W. Zhang, M. J. Serpe, Y. J. Huang and T. Chen, Adv. Funct. Mater., 2018, 28, 1704568.

19 K. Haraguchi and T. Takehisa, Adv. Mater., 2002, 14, 1120-1124. 20 L. Y. Li, F. X. Lu, C. Wang, F. L. Zhang, W. H. Liang, S. Kuga, Z. C. Dong, Y. Zhao, Y. Huang and M. Wu, J. Mater. Chem. A, 2018, 6, 24468-24478.

21 X. H. Liu, D. B. Wu, H. L. Wang and Q. G. Wang, Adv. Mater., 2014, 26, 4370-4375. 
22 Y. Ding, J. J. Zhang, L. Chang, X. Q. Zhang, H. L. Liu and L. Jiang, Adv. Mater., 2017, 29, 1704253.

23 Q. Chen, L. Zhu, C. Zhao, Q. Wang and J. Zheng, Adv. Mater., 2013, 25, 4171-4176.

24 C. W. Peak, J. J. Wilker and G. Schmidt, Colloid Polym. Sci., 2013, 291, 2031-2047.

25 Y. K. Wang, F. Chen, Z. X. Liu, Z. J. Tang, Q. Yang, Y. Zhao, S. Y. Du, Q. Chen and C. Y. Zhi, Angew. Chem., Int. Ed., 2019, 58, 15707-15711.

26 F. Mo, G. Liang, Q. Meng, Z. Liu, H. Li, J. Fan and C. Zhi, Energy Environ. Sci., 2019, 12, 706-715.

27 M. J. Earle and K. R. Seddon, Pure Appl. Chem., 2000, 72, 1391-1398.

28 T. Welton, Chem. Rev., 1999, 99, 2071-2083.

29 L. Crowhurst, N. L. Lancaster, J. M. P. Arlandis and T. Welton, J. Am. Chem. Soc., 2004, 126, 11549-11555.

30 S. Hong, H. L. Lian, M. Z. Pan and L. Chen, BioResources, 2017, 12, 3017-3029.

31 P. Wang, S. M. Zakeeruddin, I. Exnar and M. Gratzel, Chem. Commun., 2002, 2972-2973.

32 M. C. Buzzeo, R. G. Evans and R. G. Compton, ChemPhysChem, 2004, 5, 1106-1120.

33 Y. J. Kang, H. Chung, C.-H. Han and W. Kim, Nanotechnology, 2012, 23, 065401.

34 Y. J. Kang, S.-J. Chun, S.-S. Lee, B.-Y. Kim, J. H. Kim, H. Chung, S.-Y. Lee and W. Kim, ACS Nano, 2012, 6, 6400-6406.

35 D. Mecerreyes, Prog. Polym. Sci., 2011, 36, 1629-1648.

36 J. Yuan and M. Antonietti, Polymer, 2011, 52, 1469-1482.

37 M. M. Obadia, B. P. Mudraboyina, A. Serghei, D. Montarnal and E. Drockenmuller, J. Am. Chem. Soc., 2015, 137, 6078-6083.

38 L. Balo, Shalu, H. Gupta, V. K. Singh and R. K. Singh, Electrochim. Acta, 2017, 230, 123-131.

39 M. Amjadi, A. Pichitpajongkit, S. Lee, S. Ryu and I. Park, ACS Nano, 2014, 8, 5154-5163.

40 A. P. Abbott, D. Boothby, G. Capper, D. L. Davies and R. K. Rasheed, J. Am. Chem. Soc., 2004, 126, 9142-9147.

41 F. Del Monte, D. Carriazo, M. C. Serrano, M. C. Gutierrez and M. L. Ferrer, ChemSusChem, 2014, 45, 999-1009.

42 E. L. Smith, A. P. Abbott and K. S. Ryder, Chem. Rev., 2014, 114, 11060-11082.

43 J. D. M. Morales, R. J. Sánchez-Leija, A. Carranza, J. A. Pojman, F. D. Monte and G. Luna-Bárcenas, Prog. Polym. Sci., 2018, 78, 139-153.

44 P. Zhu, Z. Gu, S. Hong and H. Lian, Carbohydr. Polym., 2017, 177, 217-223.

45 A. Abo-Hamad, M. Hayyan, M. A. AlSaadi and M. A. Hashim, Chem. Eng. J., 2015, 273, 551-567.

46 C. Mukesh, R. Gupta, D. N. Srivastava, S. K. Nataraj and K. Prasad, RSC Adv., 2016, 6, 28586-28592.

47 H.-R. Jhong, D. S.-H. Wong, C.-C. Wan, Y.-Y. Wang and T.-C. Wei, Electrochem. Commun., 2009, 11, 209-211.

48 Y. J. Ju, C. H. Lien, K. H. Chang, C. C. Hu and D. S. H. Wong, J. Chin. Chem. Soc., 2012, 59, 1280-1287.

49 M. Sharma, C. Mukesh, D. Mondal and K. Prasad, RSC Adv., 2013, 3, 18149-18155.
50 C. Mukesh, D. Mondal, M. Sharma and K. Prasad, Carbohydr. Polym., 2014, 103, 466-471.

51 S. Hong, Y. Yuan, Q. Yang, P. Zhu and H. Lian, Carbohydr. Polym., 2018, 201, 211-217.

52 J. A. Sirvio, M. Visanko and H. Liimatainen, Green Chem., 2015, 17, 3401-3406.

53 J. A. Sirvio, J. Mater. Chem. A, 2019, 7, 755-763.

54 Y. Liu, W. Chen, Q. Xia, B. Guo, Q. Wang, S. Liu, Y. Liu, J. Li and H. Yu, ChemSusChem, 2017, 10, 1692-1700.

55 S. Hong, H. L. Lian, X. Sun, D. Pan, A. Carranza, J. A. Pojman and J. D. Motamorales, RSC Adv., 2016, 6, 89599-89608.

56 H. Lian, S. Hong, A. Carranza, J. D. Mota-Morales and J. A. Pojman, RSC Adv., 2015, 5, 28778-28785.

57 H. Cruz, N. Jordao and L. C. Branco, Green Chem., 2017, 19, 1653-1658.

58 A. P. Abbott, R. C. Harris and K. S. Ryder, J. Phys. Chem. B, 2007, 111, 4910-4913.

59 M. X. Liu, J. D. Huang, B. H. Luo and C. R. Zhou, Int. J. Biol. Macromol., 2015, 78, 23-31.

60 C. J. Zhou, Q. L. Wu, Y. Y. Yue and Q. G. Zhang, J. Colloid Interface Sci., 2011, 353, 116-123.

61 A. R. Abbott, G. Capper and S. Gray, ChemPhysChem, 2006, 7, 803-806.

62 M. F. Zhu, Y. Liu, B. Sun, W. Zhang, X. L. Liu, H. Yu, Y. Zhang, D. Kuckling and H. J. P. Adler, Macromol. Rapid Commun., 2006, 27, 1023-1028.

63 P. Saravanan, M. P. Raju and S. Alam, Mater. Chem. Phys., 2007, 103, 278-282.

64 C. C. Chen, H. Y. Wang, S. Y. Li, L. Fang and D. G. Li, Cellulose, 2017, 24, 5487-5493.

65 J. Yang, C.-R. Han, J.-F. Duan, M.-G. Ma, X.-M. Zhang, F. Xu, R.-C. Sun and X.-M. Xie, J. Mater. Chem., 2012, 22, 22467-22480.

66 M. S. Q. Zhu, S. S. He, Y. F. Dai, J. Han, L. H. Gan, J. Liu and M. N. Long, ACS Sustainable Chem. Eng., 2018, 6, 17087-17098.

67 Z. H. Tang, X. L. Lyu, A. Q. Xiao, Z. H. Shen and X. H. Fan, Chem. Mater., 2018, 30, 7752-7759.

68 R. Tamate, K. Hashimoto, T. Horii, M. Hirasawa, X. Li, M. Shibayama and M. Watanabe, Adv. Mater., 2018, 30, 1802792.

69 Y. Zhou, C. J. Wan, Y. S. Yang, H. Yang, S. C. Wang, Z. D. Dai, K. J. Ji, H. Jiang, X. D. Chen and Y. Long, Adv. Funct. Mater., 2019, 29, 1806220.

70 Z. Gui, H. Zhu, E. Gillette, X. Han, G. W. Rubloff, L. Hu and S. B. Lee, ACS Nano, 2013, 7, 6037-6046.

71 V. A. Kusuma, M. K. Macala, J. Liu, A. M. Marti, R. J. Hirsch, L. J. Hill and D. Hopkinson, J. Membr. Sci., 2018, 545, 292-300.

72 M. Zhu, L. Yu, S. He, H. Hong, J. Liu, L. Gan and M. Long, ACS Appl. Energy Mater., 2019, 2, 5992-6001.

73 X. H. Wang, F. Song, D. Qian, Y. D. He, W. C. Nie, X. L. Wang and Y. Z. Wang, Chem. Eng. J., 2018, 349, 588-594.

74 C. J. Zhou and Q. L. Wu, Colloids Surf., B, 2011, 84, 155-162.

75 A. Kumar, Y. S. Negi, V. Choudhary and N. K. Bhardwaj, J. Mater. Phys. Chem., 2014, 2, 1-8. 
76 J. Y. Ma, K. Fu, J. Shi, Y. J. Sun, X. X. Zhang and L. Ding, Carbohydr. Polym., 2016, 151, 565-575.

77 S. L. Liu, M. M. Kang, K. W. Li, F. Yao, O. Oderinde, G. D. Fu and L. Q. Xu, Chem. Eng. J., 2018, 334, 2222-2230.

78 S. L. Liu, O. Oderinde, I. Hussain, F. Yao and G. D. Fu, Polymer, 2018, 144, 111-120.

79 T. H. Qazi, R. Rai and A. R. Boccaccini, Biomaterials, 2014, 35, 9068-9086.

80 R. Muchakayala, S. H. Song, J. W. Wang, Y. H. Fan, M. Bengeppagari, J. J. Chen and M. L. Tan, J. Ind. Eng. Chem., 2018, 59, 79-89.

81 X. W. Zhong, J. Tang, L. J. Cao, W. G. Kong, Z. Sun, H. Cheng, Z. G. Lu, H. Pan and B. M. Xu, Electrochim. Acta, 2017, 244, 112-118.
82 C. Liu, W. Chen, S. Hong, M. Pan, M. Jiang, Q. Wu and C. Mei, Nanomaterials, 2019, 9, 405.

83 G. A. Tiruye, D. Munoz-Torrero, J. Palma, M. Anderson and R. Marcilla, J. Power Sources, 2016, 326, 560-568.

84 Y. Kumar, G. P. Pandey and S. A. Hashmi, J. Phys. Chem. C, 2012, 116, 26118-26127.

85 V. Chaudoy, F. T. Van, M. Deschamps and F. Ghamouss, J. Power Sources, 2017, 342, 872-878.

86 C. W. Liew, S. Ramesh and A. K. Arof, Int. J. Hydrogen Energy, 2014, 39, 2953-2963.

87 Q. M. Tu, L. Q. Fan, F. Pan, J. L. Huang, Y. Gu, J. M. Lin, M. L. Huang, Y. F. Huang and J. H. Wu, Electrochim. Acta, 2018, 268, 562-568. 